EESTI NSV TEADUSTE AKADEEMIA TOIMETISED. X KÖIDE

FOOSIKALIS-MATEMAATHLISTE JA TEHNILISTE TEADUSTE SEERIA, 1961, NR. 4

ИЗВЕСТИЯ АҚАДЕМИИ НАУК ЭСТОНСКОИ ССР. ТОМ Х

СЕРИЯ ФИЗИКО-МАТЕМАТИЧЕСКИХ И ТЕХНИЧЕСКИХ НАУК. 1961, № 4

\title{
О НЕКОТОРЫХ ЗАКОНОМЕРНОСТЯХ ПАДЕНИЯ ТЕЛ ВО ВЗВЕШЕННОМ СЛОЕ
}

\section{Э. СИЛЬВЕР}

В области исследования закономерностей падения тел в двухфазных средах $\mathrm{k}$ настоящему времени изучены основные явления, присущие падению тел только в гяжелых минеральных суспензиях, применяемых при гравитационном обогащенин лолезных ископаемых. Максимальный диаметр твердых взвешенных чистиц в этих суспензиях не превышает 0,1 мм, их объемное содержание - 30-35\%. В дальнейшем под термином «двухфазная среда» мы подразумеваем среду, состоящую из жидкости и взеешенных в ней тем или иным способом твердых частиц. В основном исследователи придерживаются точки зрения, согласно которой в случае, когда отнощение диаметров падающего тела и взвешенной частицы меньше некоторого критического значення, следует применять закономерности стесненного падения; если же это отношение больше критического, то закономерности падения тел в истинных жидкостях можно распространить и на падение тел в двухфазной среде. Значение критического отношения диаметров падающего тела $d_{\mathrm{T}}$ и взвешенной частиыы $d_{4}$, по Музылеву ['], равно $\left(d_{\mathrm{T}} / d_{\mathrm{q}}\right)_{\kappa р} \approx 20$, а по Верховскому и Шохину $\left[{ }^{2}\right]-\left(d_{\mathrm{T}} / d_{4}\right)_{\kappa р} \approx 5$. Эти зиаче. ния экспериментальной проверке не подвергались. В случае $\left(d_{\mathrm{T}} / d_{\mathrm{q}}\right)<\left(d_{\mathrm{T}} / d_{q}\right)_{\mathrm{\kappa p}}$ для нахождения скорости падения тела в двухфазной среде разные авторы $\left[{ }^{2,3}\right]$ рекомендуют использовать различные формулы стесненного падения. По нашему мненню, практинеское применение рекомендованных зависимостей неосуществимо, поскольку падение тела в двухфазной среде существенно отличается от падення в стесненных условиях. Все рекомендованные формулы получены в результате изучения закономерностей взвешенного слоя й позволяют определять только скорость восходящей струн кзвешивающей среды, не позволяя делать никаких выводов о поведенин в тақой средс частиц других размеров и удельного веса.

В случае распространения закпномерностей падения тел в истинных жндкостях на их падение в двухфазной среде мы сталкиваемся с трудностями при определенин вязкости и удельного веса среды. Исследование вязкости двухфазных сред показывает, что вязкость коллондных растворов зависит, помимо концентрации твердой фазы, еще и от физико-химических процессов, протекающих на поверхностях раздела фаз [ $\left.{ }^{4}\right]$. С переходом к суспензиям, то есть к двухфазным средам с более крупным; взвешенными частицами, начинают играть роль уже крупңость самих частиц и относительные движения фаз. В этой области, по-видимому, поверхностные физнко-химнческие процессы уже играют меньшую роль, сходя вообще на нет по мере увеличения размеров взвешенных частиц. До сих пор мы не обладаем достаточно надежным методом определения вязкости суспензии. Что касается удельного веса двухфазной среды, то Марголин [5] указывает, что он не всегда может быть вычислен путем суммирования масс в единице объема и что его значение во многих случаях остается неопределенным.

Экспернментальная часть настоящей работы выполнена прн Институте энергетики АН ЭССР, 
На основании этого можно заключить, что изучение закономерностей падения тел ғ двухфазных средах с более крупными взвешенными частицами, чем частнцы в тяжелых минеральных суспензиях, представляет не только теоретический интерес, но не лишено и практического значения, так как полученные сведения могут быть использөваны при решении задач химической технологии, гравитационного обогащения, 1ндротехники и т. д.

Приблизиться к решению вопросов, возникающих при изучении падения тел в двухфазных средах, по нашему мнению, можно легче всего путем изучения падения тел во взвешенном (псевдоожиженном) слое. Восходящим потоком жидкости можно поддерживать во взвешенном состоянии довольно крупные частицы. В то же время регулированне состояния взвешенного слоя в течение эксперимента осуществляется изменением одной лишь скорости восходящего потока жидкости.

Применим в качестве модели промышленного аппарата трубу диаметром $D$.* В этой трубе создадим из твердых монодисперсных частищ, диаметром $d_{4}$ взвешенный слой. Образованный восходящим со скоростью $ш$ потоком жидкости взвешенный слой имеет порозность $\varepsilon$, динамическое равновесие слоя характеризуется постоянством высоты слоя $L$. Предположим, что в этом взвешенном слое под влиянием силы тяжести падает тело диаметром $d_{\tau}$ с конечной скоростью падения $v$. Последняя будет меньше скорости падения того же тела в чистой жидкости, так как дополнительно к гидродинамическому воздействию жидкости на тело оказывают влияние восходящее движение жидкости ғ взвешенные в ней твердые частицы, увеличивающие сопротивление движению. Для вычисления конечной скорости падения тела мы не можем пользоваться уравнениями, применяемыми для этой цели в случае чистых жидкостей, так как в данном случае величины удельного веса и вязкости среды не поддаются определению. Мы полагаем, что возникающие в связи с этим затруднения можно избежать путем применения метода анализа размерностей.

Скорость падения тела во взвешенном слое, очевидно, является функцией целого ряда физических параметров падающего тела, взвешенных частиц, взвешивающей среды и геометрических условий опыта. Для нахождения этих параметров запишем дифференциальные уравнения гидродинамики для обтекания тела жидкостью

$$
\begin{gathered}
(\bar{v}, \text { grad }) \bar{v}=-\frac{1}{\varrho_{ж}} \operatorname{grad} p+\bar{g}+\frac{\mu_{ж}}{\varrho_{ж}} \nabla^{2} v \\
\operatorname{div} \bar{v}=0
\end{gathered}
$$

и, в качестве условия однозначности, дифференциальное уравнение установившегося движения тела в жидкости

$$
\frac{d v}{d t}=g\left(\frac{\varrho_{\mathrm{T}}-\varrho_{\mathrm{W}}}{\varrho_{\mathrm{T}}}\right)-c_{R} \psi\left(d_{\mathrm{T}}\right) \frac{v^{2} \varrho_{\%}}{2 \varrho_{\mathrm{T}}}=0
$$

Соответствующими исследованиями [ $\left.{ }^{6}\right]$ установлено, что коэффициент сопротивления $c_{R}$ зависит от $d_{\mathrm{T}}, \mathrm{Q}_{\mathrm{\tau}}, v, \mathrm{Q}$ ж, $\mu_{\text {ж }}$ и $g$. Краевые условия мы можем опустить, поскольку они не содержат других параметров, кроме скоростей.

Дифференциальные уравнения гидродинамики для взвешенного слоя имеют вид

$$
(\bar{w}, \operatorname{grad}) \bar{w}=-\frac{1}{\varrho_{ж}} \operatorname{grad} p+\bar{g}+\frac{\mu_{ж}}{\varrho_{ж}} \nabla^{2} w,
$$

* Список обозначений, нспользованных в работе, см. в конце статьн (стр. 300). 
к которым прибавим условие однозначности, выражающее условие взвешивания [7]

$$
\Delta p=\left(\mathrm{Q}_{4}-\mathrm{Q}_{*}\right) g L(1-\varepsilon)
$$

Краевые условия опускаем и здесь.

Нужно отметить, что необходимость применения метода анализа размерностей вызывается тем, что для случая падения тела во взвешенном слое на основании до сих пор выполненных исследовательских работ мы не сможем сформулировать условия однозначности для дифференциальных уравнений гидродинамики.

Рассматривая вышеприведенные уравнения (1), (2), (3) и (4), можно предположить, что скорость падения тела во взвешенном слое являегся функцией десяти параметров

$$
v=f\left(w, \mu_{*}, d_{\mathrm{r}}, d_{\psi}, \mathrm{Q}_{\mathrm{T}}, \mathrm{Q}_{4}, \mathrm{Q}_{*}, g, \varepsilon, L\right) .
$$

K этим параметрам необходимо прибавить еще параметр, учитывающий ограниченность пространства в модели - диаметр трубы экспериментальной установки $D$. Для полноты картины необходимо было бы дополнить перечень определяющих параметров коэффициентами формы падающего тела и взвешенной частицы. Но поскольку исследование намечено провести с помощью шарообразных падающих тел, то коэффициент формы этих тел может быть опущен. Целесообразно исключить также коэффициент формы взвешенных частиц, так как ввиду невозможности постановки опытов со строго шарообразными взвешенғыми частицами мы не в состоянии выявить влияние неправильности формы частиц на скорость падения.

По поводу общей функции можно отметить следующее. Поскольку скорость восходящей струи жидкости задана, то при нзвестных диаметре и плотности взвешенных частиц порозность слоя определяется однозначно. Ввиду этого оказывается возможным опустить и показатель порозности. Но состояние взвешенного слоя, в частности количество твердой фазы в единице объема слоя, в ряде случаев целесюобразно характеризовать порозностью. Поэтому мы решили в одном случае за исходную величину принимать скорость восходящего потока, в другом случає - порозность слоя. Далее, исследования свойств взвешенного слоя $\left[{ }^{8,9}\right]$ показывают, что высота последнего влияет на его состояние в том случае, когда показатель экспериментально определенного перепада гидродинамического давления не равняется теоретически вычисленному. В наших исследованиях экспериментальные и теоретические перепады давления расходились незначительно, ввиду чего мы считаем возможным не училывать влияние высоты слоя.

Таким образом, мы приходим к заключению, что скорость падения шаров во взвешенном слое является функцией девяти независимых переменных:

1) с учетом скорости восходящей струи

$$
v=f_{1}\left(\varrho_{\kappa}, \mu_{\kappa}, w, d_{4}, \varrho_{4}, d_{\mathrm{r}}, \mathrm{Q}_{\mathrm{r}}, \dot{g}, D\right)
$$

2) с учетом порозности взвешенного слоя

$$
v=f_{2}\left(\varrho_{\kappa}, \mu_{ж}, d_{4}, Q_{4}, d_{1}, Q_{1}, g, D, \varepsilon\right) .
$$

Предполагая, что (6) и (7) можно представить в виде степенных одночленов, составляем системы уравнений, содержащие по три урав- 
нения каждая, согласно числу независимых размерностей (сила, длина, время). Для решения необходимо каждый раз принимать шесть из неизвестных заданными, только тогда можно найти значение трех остальных неизвестных в виде функций от шести заданных. Поступая таким образом, мы получим большое количество критериальных уравнений, которые с теоретической точки зрения все равноценны. Исходя нз практических соображений, мы выбрали из них для обработки экспериментальных данных следующие два:

1) если взвешенный слой характеризуется скоростью восходящей струи, то

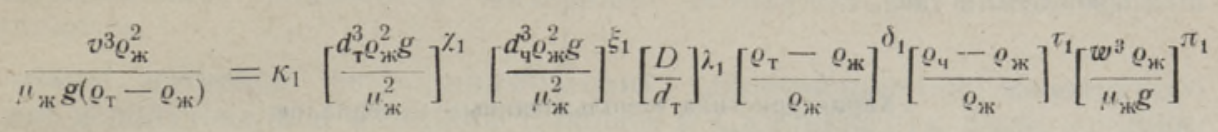

2) если взвешенный слоӥ характеризуется порозностью, то

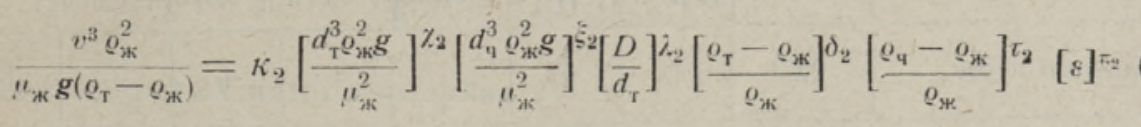

Для сокращенного написания критериальных уравнений (8) и (9) принимаем общепринятые обозначения критериев.

Критериальные уравнения вида (8) и (9) действительны, если критерин разделимы, что, как это выяснится ниже, на самом деле не так. Однако этим ценность уравнениӥ (8) и (9) не снижается, так как они указывают, какие именно критерии необходимо принять во внимание.

При экспериментировании мы пользовались трубами трех диаметров - 38, 63 и 89 мм. Общая схема экспериментальной установки показана на фиг. 1.

Падение тел происходит в трубе 1 . Поддон 3 служит одновременно опорой для трубы и местом для размещения поддерживающей взвешенный слой постели 4. Взвешивающая среда - вода подавалась по подводящему трубопроводу 7 из напорного бака 8 . Количество подаваемой воды регулировалось при помощи вентилей 6 , а перепад гидродинамического давления дифференциальным манометром 5. Датчики измерительной аппаратуры 2 были надеты на трубу так, чтобы нсключить в начальной стадин ускоренное движе ние и влияние дна аппарата на скорость падения. Расстояние между датчиками в отдельных опытах нзменялось, составляя в среднем $0,9-1,0 \mathrm{~m}$.

Нами были применены две конструкции измерительной аппаратуры. Первая, основанная на магнитных явлениях, обладала недостаточной чувствительностью. Вторая же, сконструированная физиком Э. Мартинсоном и автором, чувствительнее всех применявшихся доныне аппаратур. Она позволяла регистрировать прохождение тела с минимальным значением $d_{\mathrm{T}} / D=0,068$. Достойнством нашей конструкции является также то, что

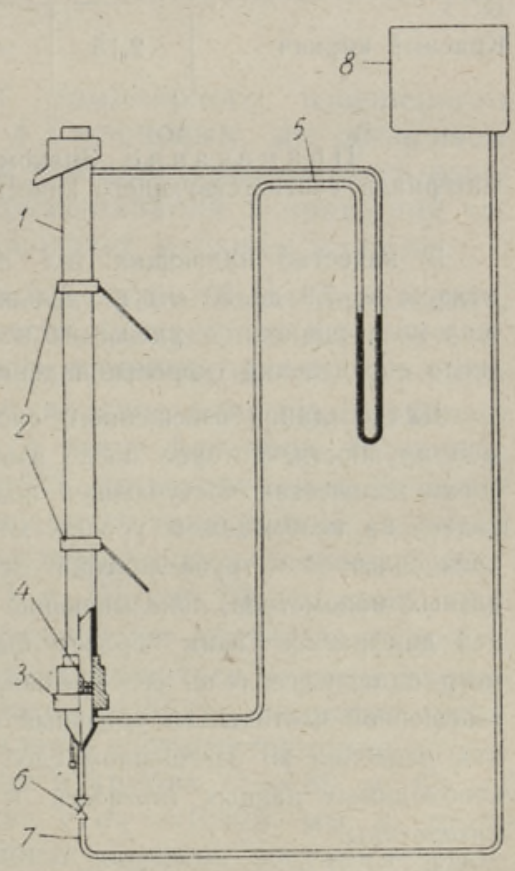

Фиг. 1. Схема экспериментальной установки. 
отпадает необходнмость изготовления специальных ферромагнитных падающих те.7. Точность измерения времени при помощи нашей аппаратуры составляет 0,01 сек.

В качестве взвешиваемых материалов мы применяли минералы, встречающиеся на территорни Эстонской ССР, а именно: 1) кварцевый песок месторождения Мяннику, 2) пирит-марказит Маардуского месторождения, 3) галенит месторождення Навести, 4) красный кирпич. Нсходные материалы дробнлись, сортировались, измельчались и классифицировались на ситах. Классы крупности характеризовались средним диаметром частиц, вычисленным как среднее арифметическое размеров отверстин̆ сит. Для каждого материала пикнометрическим способом определялся удельный вес. Качество разделения проверялось под микроскопом. Данные о взвешенных материалах прнведены в табл. 1.

таблица 1

Характеристика использованных материалов

\begin{tabular}{|c|c|c|c|c|c|c|c|c|}
\hline \multirow[b]{2}{*}{ Материал } & \multirow{2}{*}{$\begin{array}{c}\text { Удельный } \\
\text { вес, } \\
\text { r/cм }\end{array}$} & \multicolumn{7}{|c|}{ Классы крупности, мм } \\
\hline & & $\begin{array}{r}-0,179 \\
+0,087\end{array}$ & $\begin{array}{l}-0,275 \\
+0,179\end{array}$ & $\begin{array}{r}-0,375 \\
+0,275 \\
\end{array}$ & $\begin{array}{l}-0,525 \\
+0,375\end{array}$ & $\begin{array}{l}-0,676 \\
+0,525\end{array}$ & $\begin{array}{l}-1,233 \\
+0,676\end{array}$ & $\begin{array}{r}-3,000 \\
+1,233\end{array}$ \\
\hline Кварневый песок & 2,63 & + & + & + & + & + & + & + \\
\hline Пирит-марказит I & 3,15 & - & - & - & - & + & + & + \\
\hline Пирит-марказит II & 3,58 & + & + & + & - & + & + & + \\
\hline Галенит & 7,39 & + & + & + & + & - & - & - \\
\hline Красный кирпич & 2,15 & - & - & + & - & - & + & + \\
\hline
\end{tabular}

Пр име ч ание. Знаком $(+)$ отмечено наличне, знаком $(-)$ - отсутствне материала соответствующего класса крупности.

В качестве падающих тел применялись в основном стальные шарики диаметром от 7,9 до 30 мм и удельным весом $7,78 \mathrm{r} / \mathrm{cm}^{3}$. Использовались также полые железные шарики с удельным весом 4,25 и $6,00 \mathrm{r} / \mathrm{cm}^{3}$. Сравнительно небольшое число определений скорости падения было проведено и для кусков руды.

Для создания взвешенного слоя брали некоторое количество материала желаемой крупности, которое после взвешивания загружалось в трубу. Путем регулирования количества поступаюей воды верхняя граница взвешенного слоя устанавливалась на необходнмом уровне. Высота взвешенного слоя измерялась при помошн грнкрепленной к трубе линейки. Вслед за тем отсчнтывались показания дифференцнальных манометров, показывающие перепады давления во взвешенном слое и в мерной диафрагме. Таким образом были получены следующне исходные данные: диаметр падающего тела, его удельный вес, скорость падения тела, средний диаметр взвешенной частицы, ее удельный вес, скорость восходящего потока жидкости, перепад давления во взвешенном слое, порозность взвешенного слоя. Значения других необходимых данных (вязкости и удельного веса воды) брались из справочной литературы.

Результаты измерений вносились в таблицы, куда были внесены и значения критериев уравненнй (8) и (9). На основанни последних стронлись графнки и обрабатьвались результаты опытов. 
Порозность наших взвешенных слоев колебалась в пределах 0,904 0,513. Высота взвешенных слоев составляла в среднем в трубе днаметром $D=89$ мм - 160 см, в случае $D=63$ мм - 150 см и в случае $D=38$ мм - $120 \mathrm{~cm}$, что дает отношения $L / D$ соответственно $18,24,31,5$. Для каждого взвешенного слоя рассчитывалось отношение экспериментального и теоретического перепадов давлений. При этом было выяснено, что это отношение колеблется в пределах $0,90-0,96$. Закономерности в изменении этого отношения обнаружить не удалось, и мы полагаем, что отклонения обусловлены гидродинамическими процессами, происходящими во входной части нашей установки. Можно также полагать, что небольшое количество материала оставалось не взвешенным в жидкости.

При изучении гидродинамических закономерностей взвешенного слоя использовалась методика Ерковой и Смирнова $\left.{ }^{7}\right]$ и было установлено, что в случае турбулентного взвешенного слоя $\left(\mathrm{Re}_{\text {вз }}>20\right)$ наши экспериментальные данные совпадают с данными названных авторов

$$
\operatorname{Re}_{\mathrm{B} 3}=1,15\left(\mathrm{Ar}_{4} \varepsilon^{5}\right)^{0,5} \text {, }
$$

нли в принятых намн крнтериях

$$
\mathrm{Ly}_{4}=0,073\left(\mathrm{Ar}_{4} \varepsilon^{5}\right)^{0,5} \text {. }
$$

В случае же ламинарного взвешенного слоя $\left(\mathrm{Re}_{\text {вз }}<10\right)$ такого совпадения не получалось и наши взвешенные слои могут быть охарактеризованы зависимостью

$$
\operatorname{Re}_{\text {в3 }}=0,028 \operatorname{Ar}_{4} \varepsilon^{2,5} \text {. }
$$

Расхождение нашего результата для ламинарного взвешенного слоя от результата, полученного Ерковой и Смирновым, мы объясняем Блиянием формы частиц. Можно полагать, что для турбулентного взвешенного слоя в условиях интенсивного перемешивания и вращения частиц отличис формы от шарообразной оказывает меньшее влияние.

В начале измерения скоростей падения тел нужно было определить, какое именіо значение скорости из числа всех измеренных следует считать правильным. Хотя в принципе скорость падения однозначно определяется характеристиками тела и среды, но свое влияние оказывает и ряд второстепенных, трудно поддающихся учету факторов. В результате траектория падающего тела отклоняется от вертикали в большей или в меньшей степени и мы получаем для одного тела разные скорости гіадения. Многие исследователи вообще не затрагивают вопроса о подборе характерной скорости. Музылев [1] и Шохин [2] считают правильным в качестве таковой брать нанбольшее ее значение из числа всех зарегнстрированных. Мы решили воспользоваться в этом случае методом математической статистики и рассматривать скорость падения как случайную величину. Были поставлены соответствующие эксперименты падения шара $d_{\mathrm{r}}=9,6$ мм в воде в трубах $D=89$ и 63 мм и во взвешенном слое из кварцевого песка с $d_{4}=0,425$ мм в трубе $D=89$ мм. Количество определений скорости падения составило соответственно 89, 78 и 67 . В каждом случае мы пришли к одинаковому, вполне определенному результату. Для падения во взвешенном слое эти результаты представлены на фиг. 2. 
Наши исследования приводят к заключению, что колебания значений скорости уменьшаются с уменьшением диаметра трубы и увеличением размера и удельного веса падающего тела. Эти колебания могут быть описаны законом нормального распределения случайных величин.

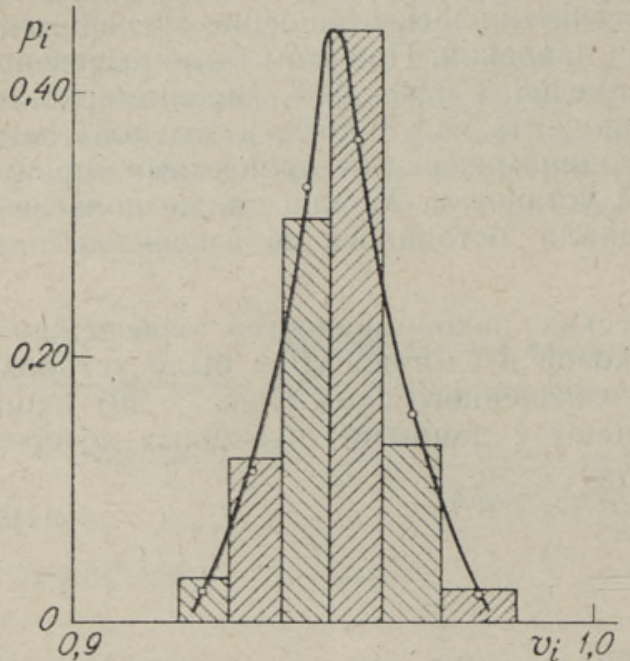

Фиг. 2. Гистограмма и выравнивающая кривая нормального распределения скорости падения шара $d_{\mathrm{T}}=9,6$ мм во взвешенном слое из кварцевого песка $d_{\mathbf{y}}=$ $=0,425$ мм в трубе $D=89$ мм.
Поэтому представляется неправильным брать в качестве основной скорости наибольшую из числа всех зарегистрированных. так как ее вероятность очень незначительна. В качестве характерной скорости следует брать наивероятнейшее еe значение, т. е. математическое ожидание. При ограниченном числе оіределений за характерную скорость следует принимать среднюю арифметическую.

Анализ наших результатов показывает, что число определений скорости для одного тела должно быть не менее пяти, причем для тел меньших размеров количество определений должно быть бо́льшим.

При исследовании падения тел во взвешенном слое оказалось, что зависимость $\mathrm{Ly}_{\mathrm{T}}$ от $\mathrm{Ar}_{\mathrm{r}}$ в исследуемой области прямолинейная, как это видно из фиг. 3. Необходимо обратить внимание на то, что при вычислении значений $\mathrm{Ly}_{\text {т }}$ в критерии входит не измеренная скорость падения, а скорость падающего тела относительно взвешивающей среды $v+w$. Из рассмотрения фиг. 3 следует еще, что $\mathrm{LV}_{\mathrm{T}}=$ $=f\left(\operatorname{Ar}_{\mathrm{\tau}}\right)$ при $D / d_{\tau}<3$ начинает отклоняться от прямолинейной ввиду воздействия стенок трубы. Мы ограничились только областью прямолинейной зависимости. Опыты показывают, что необходимо различать падение тел в ламинарном, переходном и турбулентном взве-

Фиг. 3. Зависимость $\mathrm{Ly}_{\mathrm{T}}$ от $\mathrm{Ar}_{\mathrm{T}}$ в случае ламинарного взвешенного слоя из кварцевого песка при: $1-D=89 \mathrm{мм}, 2-D=63 \mathrm{мм}$,

$$
3-D=38 \text { мм. }
$$

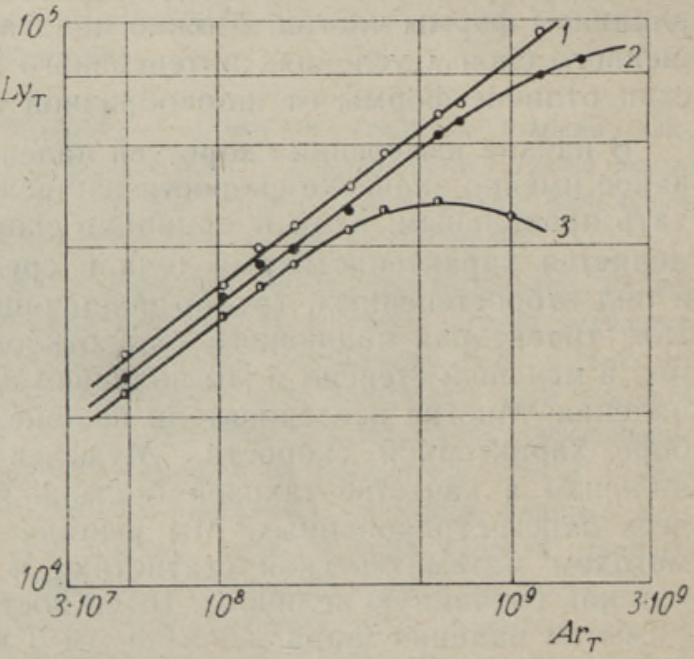

шенных слоях. К этому заключению приводит то обстоятельство, что в функции

$$
\mathrm{Ly}_{\mathrm{r}}=\kappa \mathrm{Ar}_{\mathrm{T}}^{x}
$$


в случае ламинарного взвешенного слоя $x=0,43$, а в случае турбулентного слоя $x=0,65$. В наших исследованиях не было обнаружено зависимости пристеночного эффекта от крупности взвешенной частищы.

При исследовании зависимости Lyт от порозности мы прншли к зажлючению, что

$$
-\mathrm{Ly}_{\mathrm{T}}=c \operatorname{Ar}_{\mathrm{T}}^{x} e^{y}
$$

где

$$
y=f\left(\mathrm{Ga}_{4}, \frac{\gamma_{4}-\gamma_{*}}{\gamma_{*}}\right) .
$$

Зависимость $\mathrm{Ly}_{\mathrm{\tau}}=f(\varepsilon)$ для шара с $d_{\mathrm{T}}=12,7$ мм представлена на фиг. 4 . Зависимость $\mathrm{Ly}_{\mathrm{T}}=f(\varepsilon)$ на логарифмической сетке прямолинейна и характеризуется в формуле (14) различными значениями $y$ для разных взвешенных материалов. При значении порозности $\varepsilon=1$ все прямые сходятся в одну точку, соответствующую значению $\mathrm{Ly}_{\tau}$ падения тела в чистой жидкости. Для ламинарного взвешенного слоя, характеризуемого значением критерия Рейнольдса $\mathrm{Re}_{\text {вз }}<10$, оказывается, что значение $y$

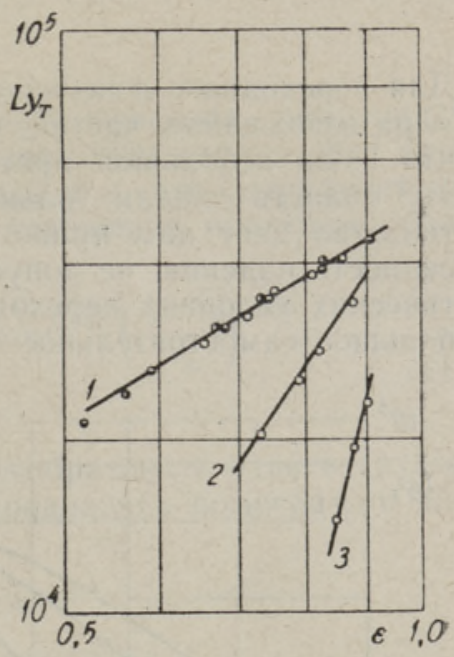

Фиг. 4. Зависимость $\mathrm{Ly}_{\mathrm{T}}$ от порозности $\varepsilon$ и удельного веса взвешенного материала: 1 - кварцевый песок $\gamma_{4}=2,63 \mathrm{r} / \mathrm{cm}^{3}$, 2 - пирит-марказит $\gamma_{4}=3,58 \mathrm{r} / \mathrm{cm}^{3}, 3-\mathrm{ra}$. ленит $\gamma_{ч}=7,39 \mathrm{r} / \mathrm{cm}^{3}$. зависит не от крупности взвешенной частицы, а лишь от удельного веса взвешенной фазы. Значение $y$ выражается

$$
y=0,75\left(\frac{\gamma_{4}-\gamma_{*}}{\gamma_{\%}}\right)
$$

или в полном внде

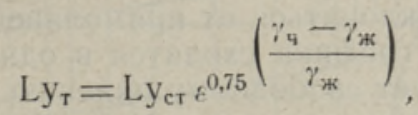

где Lycr соответствует падению тела в той же трубе в чистой жидкости.

Как было отмечено, уравнения (15) и (16) действительны для вззешенных слоев, для которых $\mathrm{Re}_{\text {вз }}<10$.

Целесообразно было бы связать скорость падения тела во взвешенном слое со скоростью падения в чистой неподвижной жидкости, сосредоточив физические параметры, учитывающие наличие и свойства твердой взвешенной фазы, в поправочный коэффициент. В уравнении (16) такая зависимость представлена в удобном виде, причем поправочным коэффициентом является величина $\varepsilon^{y}$ из уравнения (14). В дальнейшем мы и будем придерживаться такого способа представления экспериментальных данных.

Взвешенный слой с переходным гидродинамическим режимом взвешивания характеризуется значениями критерия Рейнольдса $10<\mathrm{Re}_{\text {вз }}<20$. 
Общий характер зависнмости $\mathrm{Ly}_{\mathrm{T}}=f(\varepsilon)$ для такого слоя подобен зависимости для ламинарного слоя, и согласно (14)

$$
y=1,20\left(\frac{\gamma_{4}-\gamma_{*}}{\gamma_{*}}\right)^{1,11} \text {. }
$$

Для переходного взвешенного слоя зависимость $\mathrm{Ly}$ т от $d_{4}$, т. е. от размера взвешенных частиц, установить не удалось. Объясняется это тем, что из-за небольшой протяженности переходного взвешенного слоя в эту область попали только материалы одной крупности. Это обстоятельство дает нам право пренебречь влиянием крупности частиц на скорость падения, не допуская этим большой ошибки, так как в практических условиях переходные взвешенные слон, очевидно, имеют небольшое самостоятельное значение.

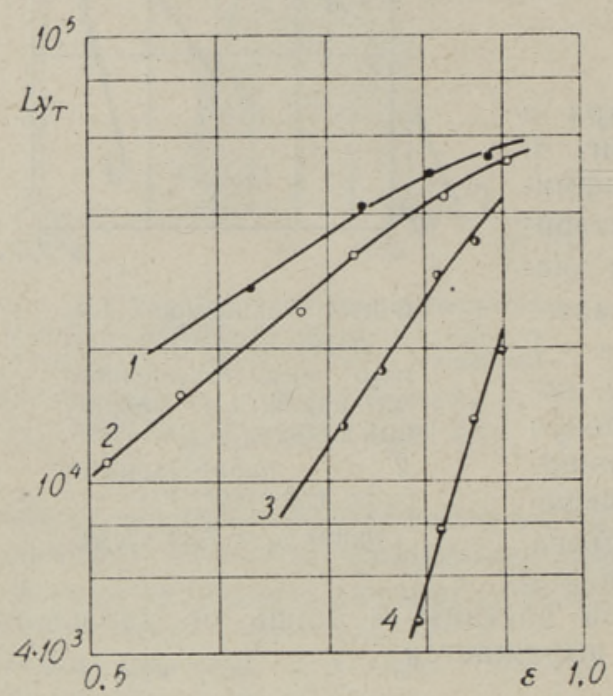

При переходе к турбулентному взвешенному слою (значение критерия $\left.\mathrm{Re}_{\text {вз }}>20\right)$ становится заметным более быстрое уменьшение критерия Лященко с уменьшением порозности слоя. Часть экспериментальных данных для турбулентного взвешенного слоя представлена на фиг. 5.

Фиг. 5. Зависимость $\mathrm{Ly}_{\mathrm{T}}$ от порозностн $\varepsilon$ для шара $d_{\mathrm{\tau}}=16,5 \mathrm{Mм}$ в турбулентном взвешенном слое из частиц $d_{4}=$ $=0,973$ мм: 1 - красный кирпич, 2 кварцевый песок, 3 - пирнт-марказит, 4 - галенит.

Легко заметить, что при значении критерия Лященко, составляюшем приблизительно $60 \% \mathrm{Ly}_{\text {т, }}$ соответствующего падению при $\varepsilon=1$, зависимость начинает отклоняться от прямолинейной. Оказывается, что прямолинейные отрезки графика сходятся в одну точку при $\varepsilon=1$, где значение $\mathrm{Ly}_{\text {т }}$ соответствует свободному падению. Отклоняющиеся ветви графнка при $\varepsilon=1$ также сходятся в одну точку, где Ly т соответствует паденню в трубе в отсутствие взвешенной фазы. Упомянутое отклонение вызвано влиянием пристеночного эффекта. Критериальное уравчение для области свободного падения выражается следующей формулой:

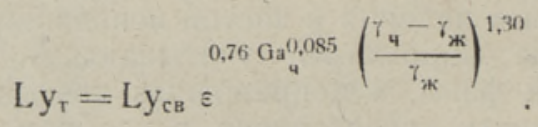

Скорость падення тела можно вычислить по формуле

$$
\lg \left(\frac{v}{v_{\mathrm{cs}}}\right)^{3}=0,76 d_{4}^{0,25} g^{0,09}\left(\frac{\gamma_{\%}}{\mu_{\%}}\right)^{0,17}\left(\frac{\gamma_{4}-\gamma_{*}}{\gamma_{*}}\right)^{1,30} \lg \varepsilon .
$$


Крнтернальное уравнение для области влияння пристеночного эффекта

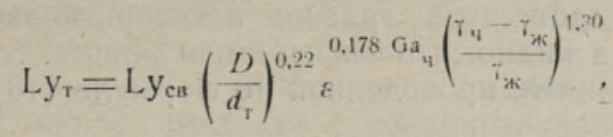

если $D / d_{\text {т }}>3$.

Для турбулентного взвешенного слоя была выведена зависимость $\mathrm{Ly}_{т}$ и от скорости восходящей струи взвешивающего потока. Эти критериальные уравнения имеют более сложный вид, что затрудняет их практическое применение, но для полноты изложения приведем уравнение для участка свободного падения

$$
\frac{L y_{\mathrm{T}}}{\mathrm{L} y_{\mathrm{CB}}}=30,2 \mathrm{Ga}_{4}^{-0,17}\left(\frac{\gamma_{4}-\gamma_{\%}}{\gamma_{\%}}\right)^{-1,60} \mathrm{Ly}_{4}{ }^{0,30 \mathrm{Ga} a_{4}^{0,085}}\left(\frac{\gamma_{4}-\gamma_{\%}}{\gamma_{\%}}\right)^{1,30} .
$$

Зная (11), можно уравнения (18) и (20) преобразовать одно в другое.

Для облегчения практнческих расчетов предлагаем номограмму для нахождення значения величнны

$$
0,76 \mathrm{Ga}^{0,685}\left(\frac{7_{4}-r_{W}}{i_{W}}\right)^{1,30}
$$

которая прнведена на фиг. 6 и позволяет проводить расчеты для свободнпго падения по формуле (18).

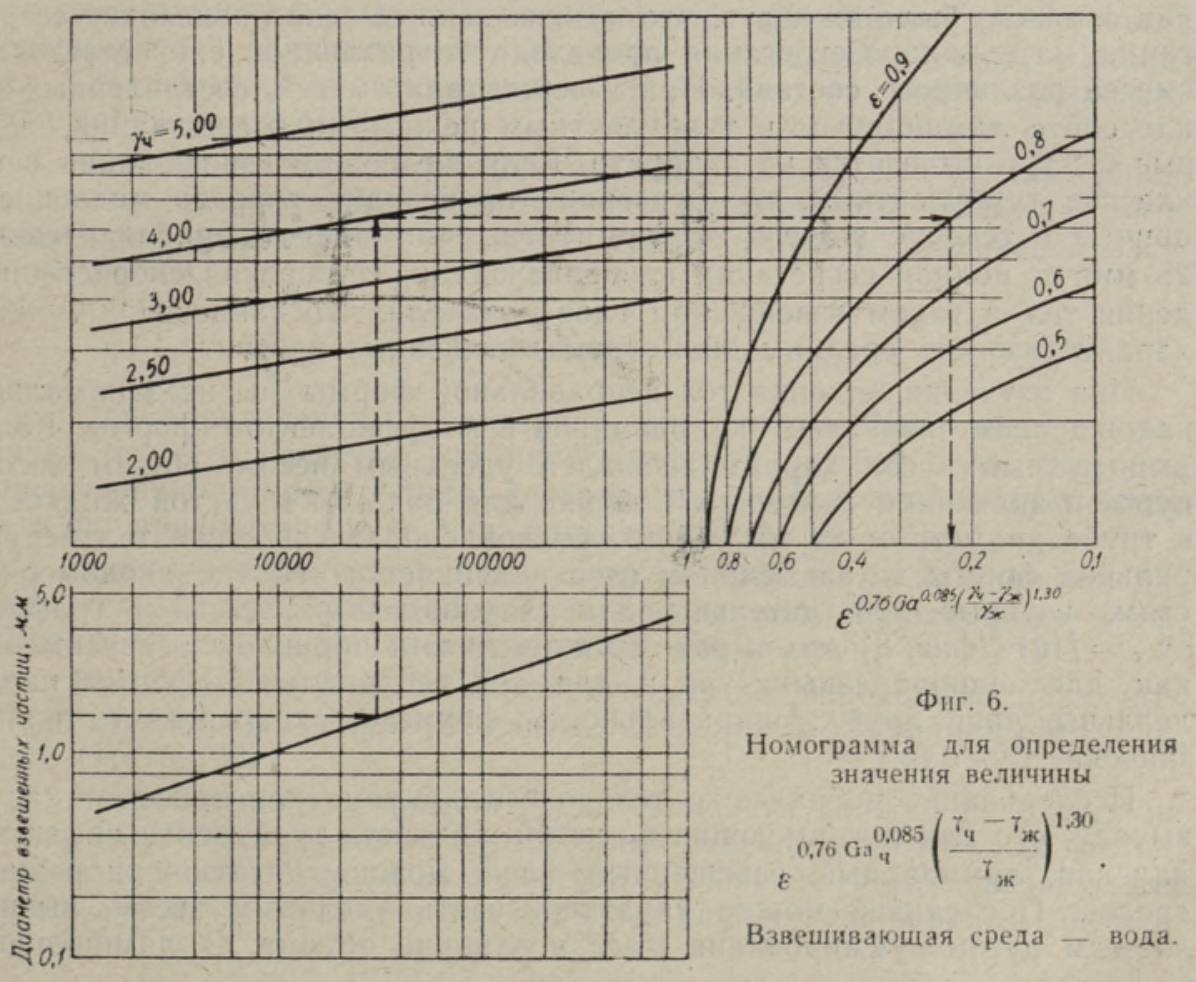


Для характеристики действительности уравнения (18) на фиг. 7 представлен корреляционный график некоторых экспериментальных данных. Из рисунка видно, что экспериментальные точки хорошо совпадают с прямой линией, проведенной по формуле (18).

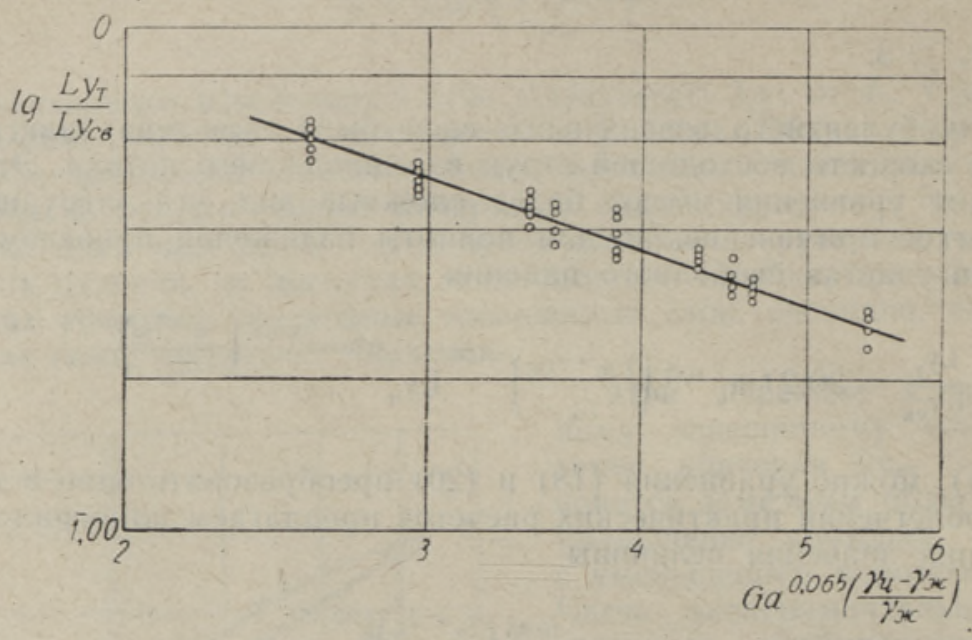

Фиг. 7. Корреляционный график падения стальных шаров диаметром 9,$6 ; 11,5 ; 12,7 ; 16,5$ и 20,5 мм в туро́улентных взвешенных слоях из кварцевого песка и пирита-марказита. Порозность 0,74 .

Дополнительно к вышеприведенному были поставлены опыты с широким диапазоном крупности взвешенных частиц. Эти опыты показали. как и можно было ожидать, что во взвешенном слое происходит сегрегация материала. Сегрегация происходит с различной скоростью для смесей различного состава. При взвешивании смесей, составленных из классов с ламинарным и турбулентным режимами взвешнвания, первые быстро выносятся из аппарата. Если же режим взвешивания всех классов турбулентный, то сегрегация происходит гораздо медленнее, причем в течение нашего эксперимента, занимавшего приблизительно 25 минут, полной сегрегации не происходило. Измерения скорости падения тел в таком взвешенном слое показали, что такие слон можно характеризовать средним диаметром взвешенных частиц.

При изучении падения тел неправильной формы мы не занимались нахождением эквивалентных размеров и коэффициентов формы. Каждый рудный кусок характеризовался удельным весом, общим весом куска и значением критерия Лященко для падения в чистой жидкости в трубе диаметром 89 мм. Было установлено, что падение тел неправильной формы во взвешенном слое подчиняется тем же закономерностям, которые действительны для шарообразных тел. На графике $\mathrm{Ly}_{\mathrm{r}}=f(y)$ (фир. 8) это выражается тем, что изображения зависимости как для шарообразных, так и для тел неправильной формы паралелльны друг другу. Неправильность формы предопределяет только значение Lут для $\varepsilon=1$.

Исследования по падению тел во взвешенном слое приводят нас $\mathrm{k}$ выводу, что для тел, имеющцих в чистой жидкости турбулентный режим падения, ламинарный взвешенный слой можно считать однородной средой. Последнюю можно характеризовать удельным весом, вычисляемым путем суммирования масс в единице объема. В ламинарном 
взвешенном слое твердые частицы, взвешенные в восходящем потоке жидкости, как бы увеличивают инерцию жидкости, что подтверждается тем, что скорости падения тела во взвешенном слое и в жидкости того же удельного веса совпадают. Очевидно, при падении тела в ламинарном взвешенном слое оно не приходит в соприкосновение со взвешенными частицами, а отделено от них слоем жидкости некоторой толщины.

Фиғ. 8. Зависимость Ly правильной формы в турбулентном взвешенном слое из кварцевого песка $d_{4}=0,973 \mathrm{мм}$ 1 - стальной шар $d_{\mathrm{T}}=9,6 \mathrm{мм}, 2-$ стальной цилиндр $10 \times 10$ мм, 3 - кусок известняка весом 53 г.

В турбулентном же взвешенном слое играют роль соударения падаюшего тела со взвешенными частицами, на что указывает то обстоятельство, что скорость падения тела во взвешенном слое значительно меньше скорости падения его в жидкости равного удельного веса. Скорость падения будет тем меньше, чем крупнее взвешенные частицы. Поэтому мы считаем, что отношение геометрических размеров

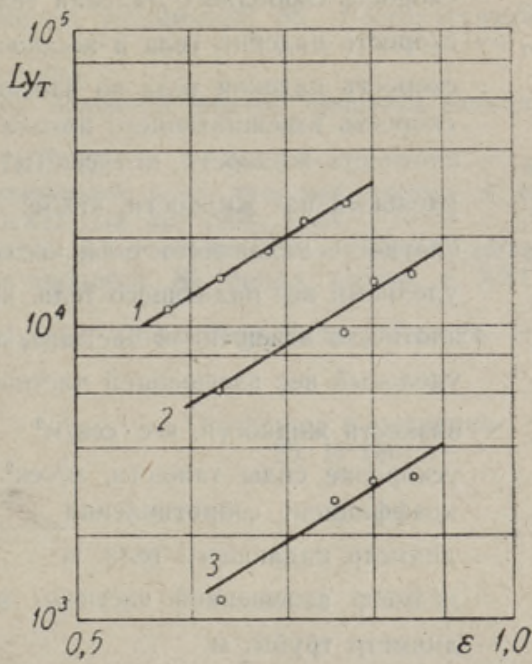
$d_{\mathrm{\tau}} / d_{\text {ч }}$ не может являться критерием перехода характера падения от одного вида падения в двухфазной среде в другой, а переход зависит от свойств самой среды, т. е. от режима обтекания взвешенных частиц. поскольку этим определяется относительное движение фаз. В наших экспериментах минимальное значение отношения $d_{\mathrm{\tau}} / d_{4}$ равнялось 3,34 , но никакой тенденции к отклонению от общей закономерности замечено не было.

В свете проведенных исследований нам кажется, что использование полученных результатов поможет более совершенному решению некоторых задач, связанных с технологической стороной применения взвешенного слоя.

\section{Выводы}

1. При определении характерной скорости падения тела неправильно брать в качестве истинной скорости наибольшую скорость, определенную во время опыта. За характерную скорость следует брать среднюю арифметическую всех определений.

2. Влияние взвешенной в жидкости твердой фазы на скорость падения тела целесообразно выразить посредством поправочного коэффициента к критериям падения в чистой жидкости.

3. При падении тела во взвешенных слоях необходимо различать слои с ламинарным и турбулентным режимами взвешивания [см. уравнения (16) и (20)].

4. Уподобление двухфазной среды жидкости должно покоиться на свойствах среды, т. е. зависеть от режима взвешивания твердых частиц, a не на значении отношения размеров падающего тела и взвешенных частиц. Уподобление допустимо только в случае ламинарного взвешенного слоя. 
5. Неправильность формы падающих тел не влияет на закономерность изменения скорости падения в зависимости от порозности слоя.

\section{Список обозначений, использованных в работе:}

$v_{\text {св }}$ - скорость свободного падения тела в жидкости, м/сек

$v_{\text {ст }}$ - скорость падения тела в жидкости в трубе, м/сек

$v$ - скорость падення тела во взвешенном слое, м/сек

w - скорость взвешивающего потока жидкости в свободном сечении трубы, м/сек

○ж - плотность жидкости, кгс $\cdot \mathrm{ce}^{2} / \mathrm{M}^{4}$

$\gamma_{\text {ж }}$ - удельный вес жидкости, кгс $/ \mathrm{m}^{3}$

$\varrho_{\text {т }}$ - плотность падающего тела, кгс $\cdot \mathrm{cek}^{2} / \mathrm{M}^{4}$

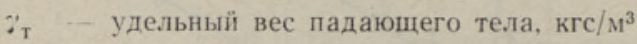

$\varrho_{4}-$ плотность взвешенной частицы, кгс $\cdot \mathrm{cek}^{2} / \mathrm{m}^{4}$

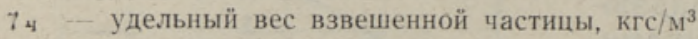

$u_{\text {ж }}$ вязкость жидкости, кгс $\cdot$ сек $/ \mathrm{M}^{2}$

$g$ - ускорение силы тяжести, м/сек ${ }^{2}$

$c_{R}$ - коэффициент сопротивления

$d_{\mathrm{T}}$ - диаметр падающего тела, м

$d_{y}$ - диаметр взвешенной частицы, м

$D$ - диаметр трубы, м

$\Delta p$ - перепад гидродинамического давлення во взвешенном слое, $\mathrm{krc} / \mathrm{m}^{2}$

$\varepsilon \quad$ - порозность взвешенного слоя

L _ высота взвешенного слоя, м

$\operatorname{Re}_{\mathrm{B} 3}=\frac{w d_{\text {ч }} \varrho_{ж}}{\mu_{ж}{ }^{\varepsilon}} \quad-$ критерий Рейнольдса для взвешенного слоя

$\mathrm{Ar}_{\mathrm{T}}=\frac{d_{\mathrm{T}}^{3} \varrho_{ж}^{2} g}{\mu_{ж}^{2}} \cdot \frac{\varrho_{\mathrm{T}}-\varrho_{ж}}{\varrho_{ж}}-$ критернй Архимеда для падающего тела

$\mathrm{Ga}_{4}=\frac{d_{ч}^{3} \varrho_{ж}^{2} g}{\mu_{ж}^{2}} \quad-$ критерий Галилея для взвешенной частицы

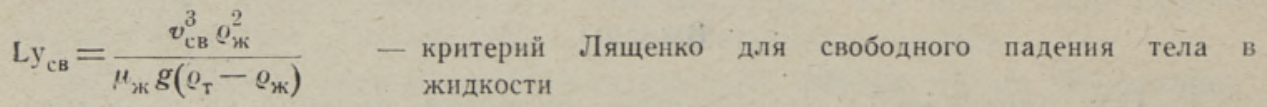
$\mathrm{Ly}_{\mathrm{c \tau}}=\frac{v_{\mathrm{c \tau}}^{3} \varrho_{\text {世 }}^{2}}{\mu_{\text {ж }} g\left(\varrho_{\mathrm{T}}-\varrho_{\text {}}\right)} \quad-$ крнтернй Лященко для падення тела в жидкости в трубе $L y_{\mathrm{T}}=\frac{v^{3} \varrho_{ж}^{2}}{\mu_{ж} g\left(\varrho_{\mathrm{T}}-\varrho_{ж}\right)} \quad-$ критернй Лященко для падения тела во взвешенном слое $\mathbf{L y} y_{4}=\frac{w^{3} \varrho_{ж}^{2}}{\mu_{ж} g\left(\varrho_{\mathrm{T}}-\varrho_{ж}\right) \varepsilon^{3}}-$ критернй Лященко для взвешенной частицы. 


\section{Л И Т Е Р А Т Р А}

1. Г. А. Музылев, Обогащение угля в минеральных суспензиях, М.. Углетехиздат, 1953.

2. Н. М. Верховский, В. Н. Шохин, О движении мннеральных зерен в суспензиях, Обогащение руд, № 6, 1958, 16.

3. С. Г. Евси ович, Обогащение руд в тяжелых суспензиях, М., Госгортехиздат, 1959.

4. Г. Р. К рой т, Наука о коллойдах, М., ИЛ, 1955.

5. И. 3. М арголин, Утяжеление среды как основной фактор процесса отсадки, Горный журнал, № 3, 1954, 44.

6. Л. П р андт ль, Гндроаэромеханика, М., ИЛ, 1951.

7. Л. Н. Ерков а, Н. И. С ми рнов, Взвешенный слой твердых частиц и его закономерности. Ж. прикл. хнмии, т. ХXIX, № 10, 1956, 1484.

8. Л. Н. Еркова, Н. И. С м и нов, Высота взвешенного слоя шарообразных тастии и ее зависимость от обстановки процесса, Ж. прикл. химии, т. XXIX, № $8,1956,1175$.

9. Д. B röt $\mathrm{z}$, Grundlagen der Wirbelschichtverfahren, Chem.-Ingr-Techn., Bd. 24, H.2, $1952,60$.
Институт химии
Академии наук Эстонской ССР
Поступила в редакцию
25. II 1961

\section{KEHADE LANGEMISE SEADUSPÄRASUSTEST HOLJUVAS KIHIS}

\section{E. Silver}

\section{Resümee}

Teaduslikus kirjanduses võib leida andmeid kehade langemisest ainult suspensioonides, kus vedelikus hōljuvate tahkete osakeste läbimc̄ōt ei ületa $0,1 \mathrm{~mm}$. Artiklis esitatakse uurimistulemusi kehade langemise kohta kahefaasilistes keskkondades, kus vedelikus hōlju. vate tahkete osakeste läbimōõt oli $0,087-3,00 \mathrm{~mm}$. Määravate tegurite dimensioonanalüü. siga on tuletatud kriteriaalvalem kehade langemise kohta hôljuvas kihis. Katseandmete läbitöötamine selle valemi alusel näitab, et hổljuvate osakeste läbimōõdust, erikaalust ja kontsentratsioonist moodustub parandustegur, mis täiendab keha vabalangemise kriteeriume. Katsetes on kasutatud torusid läbimõõduga 89,63 ja $38 \mathrm{~mm}$. Kiirused määrati spetsiaalselt ehitatud elektronaparatuuriga, mis võimaldas $89 \mathrm{~mm}$ läbimōōduga torus registreerida $7 \mathrm{~mm}$ läbimōōduga langevaid kehi, kusjuures täpsus oli 0,01 sek. Artiklis näidatakse, et tuleb eristada kehade langemist laminaarses ja turbulentses hôljuvas kihis. Laminaarses hõljuvas kihis hõljuvate osakeste suurus ei mõju langemise kiirusele; see oleneb ainult tahke faasi erikaalust ja kontsentratsioonist. Omavaheline vởrdlus näitab, et langemise kiirus laminaarses hõljuvas kihis ühtib langemise kiirusega vedelikus, mille erikaal on vōrdne hōljuva kihi erikaaluga. Viimane on arvutatud masside summeerimisega kihi ruumalaühikus. Turbulentses hôljuvas kihis seevastu on langemiskiirus palju väiksem, mis näitab osakeste ja keha kokkupõrgete mõju. On välja selgitatud, et ebakorrapärase kujuga kehade langemine hōljuvas kihis allub samadele seaduspärasustele, millele kerakujuliste kehade langeminegi. Artiklis näidatakşe, et ühe keha jaoks katseliselt määratud paljudest langemiskiirustest on õige aluseks valida määramiste aritmeetiline keskmine.

\section{Eesti NSV Teaduste Akadeemia} Keemia Instituut
Saabus toimetusse

25. II 1961 


\section{ON THE VELOCITY OF BODY FALLING IN FLUIDIZED BED}

\section{E. Silver}

\section{Summary}

Up to the present, the scientific literature provides no data as to the velocity of a body falling in a fluidized bed. The article presents some results of an investigation carried out in this branch. The size of fluidized particles was $0.087-3.00 \mathrm{~mm}$. By means of dimens ional analysis the criteria characteristic of this phenomenon were fourd. The summing up of experimental results shows that the size, specific gravity and concentration of fluidized particles compose a correction coefficient to the free falling criteria. The experiments were carried out in tubes of 89,63 and $38 \mathrm{~mm}$ in diameter and with an electronic apparatus designed for this purpose. In a laminar fluidized bed the size of fluidized particles does not affect the falling velocity of a body, which is dependent on the specific gravity and concentration of the dense phase only. The comparison of falling velocities of a body in a laminar fluidized bed and in a true liquid of the same specific gravity shows that they both coincide. In a turbulent fluidized bed the falling velocity of the same body is perceptibly smaller, which indicates the influence of the collision of the body with the fluidized particles. Bodies of irregular shape fall according to the same laws as spheres. For a body, the real falling velocity is to be computed on the basis of the arithmetical mean of velocities determined during the experiment.

Academy of Sciences of the Estonian S.S.R.,

Received Institute of Chemistry 